\title{
Stability of Janus nanoparticles at fluid interfaces $\dagger$
}

\author{
David L. Cheung ${ }^{* a}$ and Stefan A. F. Bon $* b$ \\ Received 28th April 2009, Accepted 30th June 2009 \\ First published as an Advance Article on the web 3rd August 2009 \\ DOI: $10.1039 / b 908462 b$
}

Using Monte Carlo simulations the interaction of a nanometre-sized, spherical Janus particle

(a particle with two distinct surface regions of different functionality, in this case showing amphiphilic behaviour) with an ideal fluid interface is studied. In common with previous simulations of spherical, isotropic particles, the range of the nanoparticle-interface interaction is significantly larger than the nanoparticle radius due to the broadening of the interface due to capillary waves. For a uniform particle (an isotropic particle with one surface characteristic) the stability of the particle at a liquid interface is decreased as the affinity for one liquid phase is increased relative to the other; for large affinity differences the detachment energies calculated from continuum theory become increasingly accurate. For a symmetric Janus particle (where the two different surface regions are of equal area), the presence of the particle at the interface becomes more stable upon increasing the difference in affinity between the two faces, with each face having a high affinity for the respective liquid phase. In the case studied here, where surface tension between the $A$-region of the particle with the $A$-component is identical to the surface tension between the $B$-region and $B$-component, the interaction is symmetric with respect to the nanoparticle interface separation. The particle is found to have a large degree of orientational freedom, in sharp contrast to micrometre-sized colloidal particles. Comparison with continuum theory shows that this significantly overestimates the detachment energy, due to its neglect of nanoparticle rotation; simulations of nanoparticles with fixed orientations show a considerably larger detachment energy. As the areas of the surface regions become asymmetric the stability of the Janus nanoparticle is decreased and, in the case of large differences in affinities of the two faces, the difference between detachment energies from simulation and continuum theory diminishes.

\section{Introduction}

The phenomenon of adhesion of solid particles onto liquid interfaces has been long recognised. ${ }^{1,2}$ Recently there has been a surge of interest in the behavior of nanoparticles in the vicinity of liquid-liquid interfaces. ${ }^{3}$ Much of this interest has been driven by the fact that liquid interfaces provide an attractive template for the self-assembly of nanoparticle structures, ${ }^{4,5}$ such as thin quantum dot films and cross-linked membranes ${ }^{6}$ and structures such as clusters and stripes. ${ }^{7}$ Nanoparticle interfacial self-assembly offers an attractive route to the formation of ordered nanoparticle structures for electronic, magnetic, and photonic applications. ${ }^{8}$ The adhesion of nanoparticles onto interfaces can be used to modify interfacial properties, providing a route to the formation of nanocomposite materials, including nanoparticle-stabilised foams ${ }^{9}$ and gels, ${ }^{10}$ and nanoparticle armored polymer latexes. ${ }^{11,12}$

The surface characteristics of colloidal particles, which are important for adhesion to soft interfaces, do not have to be isotropic or uniform in nature. Due to advances in the synthesis of particles, it is now possible to create particles with anisotropic

${ }^{a}$ Department of Chemistry and Centre for Scientific Computing, University of Warwick, Coventry, CV4 7AL, UK. E-mail: david.cheung@warwick.ac.uk ${ }^{b}$ Department of Chemistry University of Warwick, Coventry, CV4 7AL, UK.E-mail:s.bon@warwick.ac.uk

$\dagger$ Electronic supplementary information (ESI) available: Movies of Janus nanoparticle $\left(\alpha=90^{\circ}\right)$ with $\Delta=0.05 \sigma$ and $0.25 \sigma$. See DOI: $10.1039 / \mathrm{b} 908462 \mathrm{~b}$ "patchy" or "Janus" surface characteristics. ${ }^{13}$ Some proteins, for example fungi hydrophobin, ${ }^{14}$ also have such an amphiphilic structure. This added complexity allows self-assembly to reach a higher level, with amphiphilic Janus particles assembling into a variety of supracolloidal structures, ${ }^{15,16}$ with or without the aid of soft interfaces.

The interactions between the particle and the solvent components are fundamental in understanding and controlling the behavior of particles at fluid interfaces. Indeed, in the absence of charges, the adhesion of colloidal particles to liquid interfaces may be explained in terms of the wettability of the particle with respect to the two phases ${ }^{17}$ and expressions for the free energy of colloidal particles at interfaces may be found in terms of the associated surface tensions (and a few other quantities such as the line tension) ${ }^{17,18}$ The condition for particle stability at the interface, $\gamma_{A B} \leq\left|\gamma_{A P}-\gamma_{B P}\right|$, may also be couched in terms of the surface tensions between the particle and the solvent $\left(\gamma_{i P}, i=A, B\right)$ and the interfacial tension of the two solvent components $\left(\gamma_{A B}\right)$.

When the particle surface is anisotropic (non-uniform), existing effects, such as adhesion onto interfaces, may be amplified and new behavior may emerge. When amphiphilic Janus particles are considered, adsorption onto an interface allows both parts of the particle to be in contact with their favoured phase. Therefore, the adhesion of such particles is expected to be significantly stronger than a uniform isotropic particle, ${ }^{19,20}$ which has been verified experimentally. ${ }^{21,22}$ However, this preferred orientation of the Janus nanoparticle at the liquid-liquid 
interface may result in restricted rotational mobility, an effect not given much attention but important to take into account when fabricating supracolloidal structures.

For $\mu \mathrm{m}$-sized particles the molecular nature of the solvents may be ignored and so the effect of the particle-solvent interactions may be subsumed into macroscopic surface tensions. The molecular detail of the solvent, however, becomes important for nanoparticles (in the size range $1-10 \mathrm{~nm}$ ) and so the utility of macroscopic expressions may be questionable. This is becoming particularly relevant as, with the increasing sophistication of nanoparticle synthesis techniques, the creation of nm-scale particles with well defined surface topologies, ${ }^{23}$ with feature sizes comparable to the solvent molecules, is possible.

As such small particles are difficult to study experimentally, computer simulations provide a natural means to investigate such systems. Pioneering simulation work performed by Bresme and Quirke $24,25,26$ calculated the free energy difference between nanoparticles in bulk liquids and at fluid interfaces. These simulations suggested that macroscopic expressions may be used to describe the stability of nanoparticles at fluid interfaces, if the surface tensions are allowed to vary with particle size. However, this work only considered the free energy difference between the bound and unbound state and provided no information on the interaction between the nanoparticle and the interface. Recent simulations ${ }^{27}$ found that the nanoparticleinterface interaction was both longer-ranged and softer than predicted. This is in agreement with theoretical work ${ }^{28}$ which found that inclusion of capillary waves into a macroscopic description leads to a widening of the potential well of a particle near an interface. Simulations and liquid state theory has also recently shown that capillary waves also have a large effect on the interactions between nanoparticles adsorbed on a liquid-vapour interface. ${ }^{29}$

The aim of this paper is to study the interaction between a Janus nanoparticle, illustrated in Fig. 1 and a model fluid interface, and in particular how this is controlled by changing the difference in affinities between the two surface regions and their relative sizes. Macroscopic continuum theories predict that the stability of the particle at the interface is enhanced, which has been inferred from experiment. ${ }^{21,22}$ By calculating the free energy profiles of Janus particles at interfaces the detachment energy of

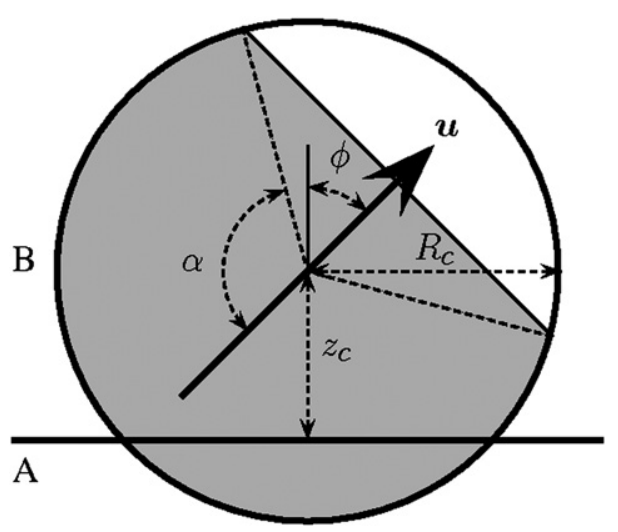

Fig. 1 Schematic representation Janus nanoparticle. $A$-philic region denoted by light gray area. the nanoparticles may be directly calculated and compared to theoretical predictions.

\section{Simulation model and methodology}

The solvent is modelled as a two-component Widom-Rowlinson (WR) fluid, ${ }^{30}$ with the interaction between two solvent molecules of species $\alpha, \beta=A, B$ given by

$$
u(r)=\left(1-\delta_{\alpha \beta}\right) u_{\mathrm{HS}}(r)
$$

where $u_{\mathrm{HS}}(r)$ is the hard sphere potential (for diameter $\sigma$ ) and $\delta_{i j}$ is the Kronecker delta function. Above a critical density this system exhibits a demixing transition which makes it an attractive model for studying interfacial phenomena despite its simplicity. ${ }^{31,32,33}$ It should be noted that due to its simplicity this model neglects many effects, such as dispersion or electrostatic interactions, that are present in experimental systems. However, this choice of this model allows us to isolate the effect of interfacial and capillary forces.

The nanoparticle-solvent interaction is also a hard-sphere like potential, with the radius depending on the solvent particle species and the orientation of the nanoparticle. Explicitly it is given by

$$
\begin{aligned}
& R_{A}= \begin{cases}R_{c}-\Delta & \hat{\boldsymbol{r}}_{i P} \cdot \boldsymbol{u}>\cos \alpha \\
R_{c}+\Delta & \hat{\boldsymbol{r}}_{i P} \cdot \boldsymbol{u}<\cos \alpha\end{cases} \\
& R_{B}=\left\{\begin{array}{cc}
R_{c}+\Delta & \hat{\boldsymbol{r}}_{i P} \cdot \boldsymbol{u}>\cos \alpha \\
R_{c}-\Delta & \hat{\boldsymbol{r}}_{i P} \cdot \boldsymbol{u}<\cos \alpha
\end{array}\right.
\end{aligned}
$$

where $\hat{\boldsymbol{r}}_{i P}$ is the separation vector between the solvent molecular and the nanoparticle, $\alpha$ defines the boundary between the $A$ and $B$-philic regions, and $R_{c}=2.5 \sigma$ is the nanoparticle radius (Fig. 1). The parameter $\Delta$, which gives the difference in radii for $A$ and $B$ particles in the range $\Delta=0$ to $\Delta=0.25 \sigma$. When $\Delta \neq 0$ there is then a region of the nanoparticle surface where one of the fluid components is excluded, which leads to a net affinity for the other component on that region. This corresponds to changing the surface tension between the nanoparticle and the solvent components. Experimentally, this may be controlled through changing attached ligands. While this study assumes a spherical nanoparticle, nanoparticles in experimental systems are often far from spherical (for example Au quantum dots are cube octahedrons). The methodology used in this work, however, may be straightforwardly extended to these more complex particle geometries. Many experimental nanoparticles also have grafted flexible chains (e.g. for stabilising particles against flocculation), which may give rise to an approximately spherical shape.

The system is studied using grand-canonical (constant- $\mu V T$ ) Monte Carlo (GCMC) simulations. For this model, temperature is not a significant phase variable (and is set to $k_{\mathrm{B}} T=1 / \beta=1$ ) and the phase behaviour is controlled by the chemical potential $\mu$ (or equivalently the density). In order to localize the interface near the cell centre, the system is confined between two walls in the $z$-direction, with periodic boundaries in the $x$ and $y$ directions. Simulations were performed at solvent chemical potential $\beta \mu=0.15$, well above the demixing transition $\left(\beta \mu_{c} \approx 0.04\right)$. The 
simulation cell dimensions were $L_{x}=L_{y}=15 \sigma$ (parallel to interface) and $L_{z}=30 \sigma$ (normal to interface). Previous work has shown that free energy profiles and binding free energies show only a weak dependence on the transverse box dimensions, ${ }^{27}$ so only one box size is studied here.

The free energy profile (or potential of mean force, PMF) for the nanoparticle is found using $\beta F\left(z_{c}\right)=-\ln P\left(z_{c}\right)$, where $P\left(z_{c}\right)$ is the probability distribution and $z_{c}$ is the nanoparticle-interface separation. The position of the interface, $z_{0}$, is found from maximizing the function

$$
f\left(z_{0}\right)=\sum_{i} q_{i} \cos \left\{\left(\frac{2 \pi}{L}\right)\left(z_{i}+z_{0}\right)\right\}
$$

where $q_{i}=1$ for $A$ and $q_{i}=-1$ for $B .^{31} z_{0}$ and $z_{c}$ are updated after every MC move. It should be noted that this gives the average interface position across the simulation cell. In order to sample $P(z)$ effectively the $z$-separation is divided into a set of overlapping windows of width $2 \sigma$. Within each window a weighting function $w(z)$, determined iteratively using the Wang-Landau algorithm, ${ }^{34}$ is applied to give a flat distribution. $w(z)$ was determined over up to $5 \times 10^{6}$ MC sweeps. Each MC sweep consists of 12125 trial moves chosen at random, with on average 6750 attempted translations (split equally between solvent molecules and the nanoparticle), 3375 attempted nanoparticle rotations, 500 attempted particle identity swaps and 500 attempted insertions and deletions, and 500 attempted nanoparticle flips $(\boldsymbol{u} \rightarrow-\boldsymbol{u})$, with move types chosen at random. The acceptance criterion for each of these different move types are outlined in the Appendix. The weight function is taken to be converged once the root mean squared deviation between successive Wang-Landau iterations is less then $0.01 k_{\mathrm{B}} T$. For each window a (weighted) probability distribution $P_{\text {sim }}(z)$ is found from a production run of $2 \times 10^{6} \mathrm{MC}$ sweeps, divided into 4 subruns. The standard deviation between the PMFs calculated for each subrun, is of the order of $0.1 k_{\mathrm{B}} T$, which is significantly larger than the convergence error (above). The true probability distribution for each window may be recovered from $P(z)=P_{\text {sim }}(z) \exp [\beta w(z)]$, and the full distribution is found using the weighted-histogram analysis method. ${ }^{35}$ The use of WangLandau sampling to calculate free energy profiles and related quantities is long established ${ }^{36}$ and has been applied in a wide variety of contexts. ${ }^{37,38}$ Despite this success a number of shortcomings have been identified, with the two main being (i) the lack of detailed balance and (ii) the saturation of error (on increasing simulation time, the accuracy of the calculation does not improve). For the latter it has been shown that the final error is of the order of $\sqrt{\ln f},{ }^{39}$ where $f$ is the WL modification factor. ${ }^{34}$ In this work, $\sqrt{\ln f}<10^{-3} k_{\mathrm{B}} T$, which, again, is substantially smaller than the variance between different subruns. For the former, it has been shown that as the modification factor becomes smaller the Wang-Landau algorithm asymptotically obeys detailed balance, which is sufficient for it to be a viable MC scheme. ${ }^{40} \mathrm{In}$ this work, once a sufficiently converged weight function is determined, the simulation is continued without updating the weight function and the probability distributions gathered from this part of the simulation. In common with other methods for determining free energy barriers it is likely to become inefficient for very large free energy barriers $\left(\approx 100 k_{\mathrm{B}} T\right)$, which may be
Table 1 Calculated nanoparticle-solvent surface tensions. Uncertainties in final digits shown in parentheses

\begin{tabular}{llrl}
\hline$\Delta / \sigma$ & $\beta \sigma^{2} \gamma_{A P}$ & $\beta \sigma^{2} \gamma_{B P}$ & $\beta \sigma^{2} \gamma_{A P}-\beta \sigma^{2} \gamma_{B P}$ \\
\hline 0.00 & $-0.04(2)$ & $-0.03(2)$ & $-0.00(3)$ \\
0.05 & $-0.06(2)$ & $0.00(3)$ & $-0.06(3)$ \\
0.10 & $-0.08(2)$ & $0.01(2)$ & $-0.10(3)$ \\
0.15 & $-0.10(2)$ & $0.04(2)$ & $-0.14(3)$ \\
0.20 & $-0.12(2)$ & $0.05(2)$ & $-0.17(3)$ \\
0.25 & $-0.15(2)$ & $0.06(2)$ & $-0.21(3)$ \\
\hline
\end{tabular}

encountered for very large particles. As Monte Carlo methods are inherently serial they become inefficient for large systems (which are impractical for single-processor calculations); for these cases, molecular dynamics simulations, in combination with methods such as metadynamics, ${ }^{41}$ may be employed.

For comparison with continuum theory, the nanoparticlesolvent surface tensions have been calculated. These were found using the method of Bresme and Quirke $e^{24,25,26}$ (which is conceptually similar to method three of Ref. 42), and follows from the free energy change associated with a change in the particle radius

$$
\mathrm{d} F=\left(8 \pi R_{c} \gamma\right) \mathrm{d} R+\left(4 \pi R_{c}^{2} P\right) \mathrm{d} R
$$

where $P$ is the pressure. A nanoparticle is simulated in a homogeneous system, with the composition taken to be the same as a WR mixture far from the interface. The free energy change may be estimated through reversibly changing the nanoparticle radius

$$
\beta \Delta F=-\ln \langle\exp (-\beta[U(R+\Delta R)-U(R)])\rangle_{R}
$$

where $U(R)$ is the free energy of a nanoparticle of radius $R$. For the present model this corresponds to counting the number of overlaps generating by increasing the nanoparticle radius. Calculating $\Delta F$ for a range of $\Delta R=0.01-0.05 \sigma$ and, as $\mathrm{d} F=\lim _{\Delta R \rightarrow 0} \Delta F$ and assuming that $\gamma$ is a slowly varying function of $R$, extrapolating to 0 yields the surface tensions. The calculated values are listed in Table 1 .

\section{Results}

In this paper we study the interaction between a spherical Janus nanoparticle, illustrated in Fig. 1, and a model fluid-interface, and in particular how this is controlled by changing the size of the two surface regions and the affinities of these regions to the solvent components. We present and discuss first the results for a homogeneous nanoparticle, corresponding to $\alpha=180^{\circ}$ in Fig. 1 . We then present results for Janus particles, first the symmetric case $\left(\alpha=90^{\circ}\right)$ followed by the asymmetric $\left(90^{\circ}<\alpha<180^{\circ}\right)$. Detachment energies calculated from simulation are compared to predictions of continuum theories, in order to assess their accuracy for nm-sized Janus particles.

\subsection{Homogeneous nanoparticles}

Shown in Fig. 2(a) are the free energy profiles for the homogeneous nanoparticle $\left(\alpha=180^{\circ}\right)$, for $\Delta$ in the range 0 to $0.25 \sigma$. In common with previous simulation work $^{27}$ the range of the 
(a)

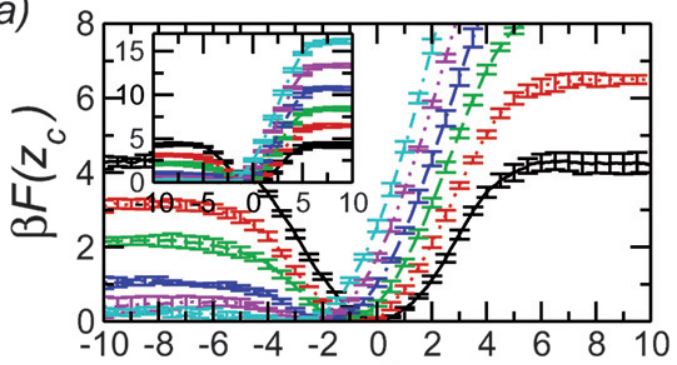

(b)

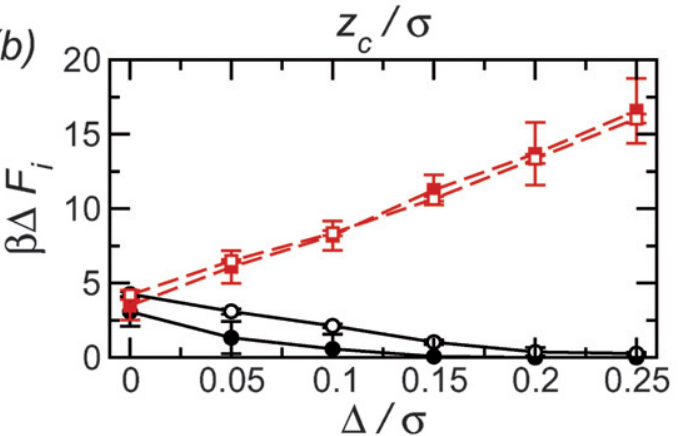

Fig. 2 (a) $\langle\cos \phi\rangle$ against nanoparticle-interface separation for Janus nanoparticle with $\Delta=0.05 \sigma$ (solid line, black), $\Delta=0.10 \sigma$ (dotted line, red), $\Delta=0.15 \sigma$ (dashed line, green), $\Delta=0.20 \sigma$ (dot-dashed line, blue) and $\Delta=0.25 \sigma$ (double-dot-dashed line, magenta). Inset shows $\langle\phi(0)\rangle$ against $\Delta$. (b) Probability maps $P(z, \cos \phi)$ for $\Delta=0.05 \sigma$ (top), $0.10 \sigma$ (middle), and $0.25 \sigma$ (bottom). (c) $P$ ( $\cos \phi$ ) for $z=0$ (top) and $z=-10 \sigma$ (bottom). Symbols as in (a).

nanoparticle-interface interaction is significantly larger than $R_{c}$, in contrast to macroscopic expressions for which the interaction range is strictly equal to the nanoparticle radius. The potential found from simulation is also softer than that from macroscopic expressions. The differences between the simulation and predicted interaction potentials are likely due to the neglect of capillary waves in the macroscopic theories. ${ }^{28,27}$ For $\Delta \neq 0$ the free energy profile is asymmetric, with the degree of asymmetry increasing with $\Delta$. As has been previously noted, ${ }^{19}$ despite not being amphiphilic such particles are often surface-active (adhere to the interface). The detachment energies $\Delta F_{A / B}$ (i.e. for entry into the $A$ and $B$-rich regions) are shown in Fig. 2(b). $\Delta F_{B}$ increases roughly linearly with $\Delta$. While $\Delta F_{A}$ decreases with $\Delta$, it remains positive for all $\Delta$ studied. This indicates that even for nanoparticles with very asymmetric interactions there is a potential minimum near the interface. For $\Delta \geq 0.20 \sigma, \Delta F_{a}<k_{\mathrm{B}} T$, so the nanoparticle is only very weakly bound to the interface. The position of the energy minimum $\left(z_{c}{ }^{\mathrm{min}}\right)$ moves deeper into the $A$-region ( $z_{c}{ }^{\min }$ decreases) as $\Delta$ increases, and for $\Delta=0.25 \sigma z_{\min }<$ $-R_{c}$, showing, again, that the interaction range is larger than $R_{c}$. For large values of $\Delta$ the particle may rapidly detach from the interface (and diffuse into the bulk of the $A$-component), similar to behaviour seen for smaller nanoparticles in the case of identical interactions between the nanoparticle and each solvent component. ${ }^{27}$ Detachment may also occur for smaller values of $\Delta$, as well as for the Janus particles studied in the following sections, although the detachment probability is lower in both these cases. It should be noted that this detachment does not affect the calculation of the PMF (indeed a small PMF at large $z_{c}$ implies rapid detachment is more probable) as $P\left(z_{c}\right)$ (hence the free energy profile) is calculated for a series of overlapping windows, with the nanoparticle confined within each of these.

The detachment energy from Pieranski theory ${ }^{17,18}$ (neglecting line tension) is given by

$$
\begin{aligned}
\Delta F_{A / B}= & -\pi R_{c}^{2} \gamma_{A B} \mp 2 \pi R_{c}^{2}\left(\gamma_{A P}-\gamma_{B P}\right) \\
& +\pi R_{c}^{2} \frac{\left(\gamma_{A P}-\gamma_{B P}\right)^{2}}{\gamma_{A B}}
\end{aligned}
$$

As shown in Fig. 2(b) the barrier for entry into the $A$-phase is consistently underestimated by eqn (7), due to its neglect of line tension and capillary waves. ${ }^{27}$ For $\Delta>0.20 \sigma$ and $\gamma_{B P}-\gamma_{A P}>\gamma_{A B}$ (for this system $\beta \sigma^{2} \gamma_{A B}=0.167$ ), ${ }^{27}$ continuum theory predicts that the particle becomes destabilised from the interface (the free energy barrier for entry into the $A$-region is 0 ). From simulation, such particles are still bound to the interface, albeit very weakly (with $\beta \Delta F_{A}<1$ ). In contrast, good agreement is found between simulation and Pieranksi theory for $\Delta F_{B}$. While this may be due to the larger effective radius of the particle in the $B$-rich region, even for the largest value of $\Delta, R_{B}=2.75 \sigma$ which is still smaller than radii for which Pierankski theory has been shown to be inaccurate. ${ }^{27}$ Alternatively, as $\left|\gamma_{A B}-\gamma_{B P}\right|$ increases this term becomes the dominant contribution to the free energy barrier.

\subsection{Symmetric Janus nanoparticles}

The free energy profile for a Janus particle (with $\alpha=90^{\circ}$ ) is shown in Fig. 3(a). On increasing $\Delta$, the barrier height increases [Fig. 3(b)], from $\beta \Delta F \approx 4.3$ for the homogeneous nanoparticle to $\beta \Delta F \approx 7.4$ for $\Delta=0.25 \sigma$. The variation in the free energy about the minimum is more rapid, indicating that there is a stronger restoring force on the nanoparticle. As observed for the homogeneous nanoparticles, the range of interaction is significantly larger the nanoparticle radius. The interaction range, however, remains roughly constant and the shape of the free energy profile is the same for all $\Delta$.

Theoretical analysis of a $\mu \mathrm{m}$-sized Janus particle predicts that the detachment energy is given by ${ }^{19,43}$

$$
\begin{aligned}
\Delta F_{A}= & \pi R_{c}^{2}\left[\gamma_{A B} \sin ^{2} \alpha+2\left(\gamma_{A P(B)}-\gamma_{B P(B)}\right)\right. \\
& \times(1+\cos \alpha)] \\
\Delta F_{A}= & \pi R_{c}^{2}\left[\gamma_{A B} \sin ^{2} \alpha+2\left(\gamma_{B P(A)}-\gamma_{A P(A)}\right)\right. \\
& \times(1-\cos \alpha)]
\end{aligned}
$$

where $\gamma_{i P(j)}$ is the surface tension of the $i$-philic half with the $j$ component. More details of the derivation of these expressions are given in the Appendix. For the present system, we take $\gamma_{A P(A)}=\gamma_{B P(B)}=\gamma_{A P}$ and $\gamma_{A P(B)}=\gamma_{B P(A)}=\gamma_{B P}$, where $\gamma_{i P}$ are calculated for a homogeneous particle. The values of these surface tensions are given in Table 1, with the difference between them increasing with $\Delta$. The detachment energies, calculated using surface tensions calculated for the homogeneous particle, are shown in Fig. 3(b). Apart from for the homogeneous $(\Delta=0)$ particle, these overestimate the detachment energy, with the difference becoming larger for increasing $\Delta$, with the difference being over $50 \%$ for $\Delta=0.25 \sigma(\beta \Delta F=7.4$ and 11.6 from simulation and theory, respectively). 

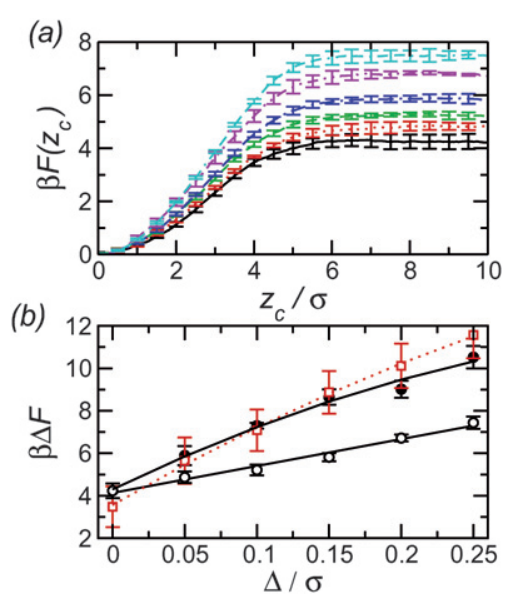

Fig. 3 (a) Free energy profiles for homogeneous nanoparticles with $\Delta=0$ (solid line, black), $\Delta=0.05 \sigma$ (dotted line, red), $\Delta=0.10 \sigma$ (dashed line, green), $\Delta=0.15 \sigma$ (dot-dashed line, blue), $\Delta=0.20 \sigma$ (double-dotdashed line, magenta), and $\Delta=0.25 \sigma$ (dot-double-dashed line, cyan). (b) Free energy barrier against $\Delta$. Squares show barrier for the $A$ component, circles show barrier for $B$ component. Open symbols denote simulation results, filled symbols from eqn (7).

The overestimation of the detachment energy by theory may arise from the assumption that the particle is oriented with the $A(B)$-philic part in the $A(B)$ phase. For large Janus particles $(>100 \mathrm{~nm})$ this is often a good assumption, ${ }^{44}$ although, as shown by Weaver et al. ${ }^{45}$ surface roughness and other effects may alter this behaviour. Nanometre-sized particles may be expected to have significantly more orientational freedom (the free energy difference between the $\mathbf{u}=\hat{\mathbf{z}}$ and $\mathbf{u}=-\hat{\mathbf{z}}$ for a particle at the interface is proportional to $\left.R_{c}{ }^{2}\right){ }^{45}$ This orientation freedom is demonstrated in Fig. 4(a), which shows $\cos \phi=\mathbf{u . \hat { z }}$ against separation. Far from the interface $\langle\cos \phi\rangle=0$, consistent with essentially random orientation of the nanoparticle. When the particle is near to the interface $\langle\cos \phi\rangle>0$ showing that the particle has a tendency to orient near the interface. As with the free energy profile, the tendency of the nanoparticle to align near the interface normal extends beyond the nanoparticle radius. This tendency becomes stronger as $\Delta$ increases, which is shown by the change in $\langle\phi(0)\rangle$, i.e. the angle between the nanoparticle orientation and $z$ (interface normal) at $z_{c}=0$, which decreases with $\Delta$. This may also be seen visually from movies of the simulation (available as ESI); $\dagger$ these show that the $\Delta=0.05 \sigma$ nanoparticle rotates more rapidly (and freely) than the $\Delta=0.25 \sigma$ particle. However, even for $\Delta=0.25 \sigma$ the coupling is still relatively weak and $\langle\phi(0)\rangle \approx 40^{\circ}$.

More information on the orientations of the nanoparticles may be seen from the probability distribution $P\left(z_{c}, \cos \phi\right)$ [Fig. 4(b)], which shows the probability of the nanoparticle having a position $z_{c}$ and orientation (relative to $\hat{\mathbf{z}}$ ) $\phi$. For all the Janus particles Near the interface $\left(\operatorname{small} z_{c}\right.$ ) this has a peak at $\cos \phi=1$, which becomes larger at $\Delta$ increases (the orienting effect becomes stronger) and the distribution becomes narrower, which may be seen from the slice through $P\left(z_{c}, \cos \phi\right)$ at $z_{c}=0$ [Fig. 4(c)]. The free energy difference between $\mathbf{u}=\hat{\mathbf{z}}$ and $\mathbf{u}=-\hat{\mathbf{z}}$ may be estimated from $\log P\left(z_{c}=0, \cos \phi=-1\right)-\log$ $P\left(z_{c}=0, \cos \phi=1\right)$; for $\Delta=0.05 \sigma$ the free energy difference is approximately $2.2 k_{\mathrm{B}} T$, which increases to approximately $6.0 k_{\mathrm{B}} T$
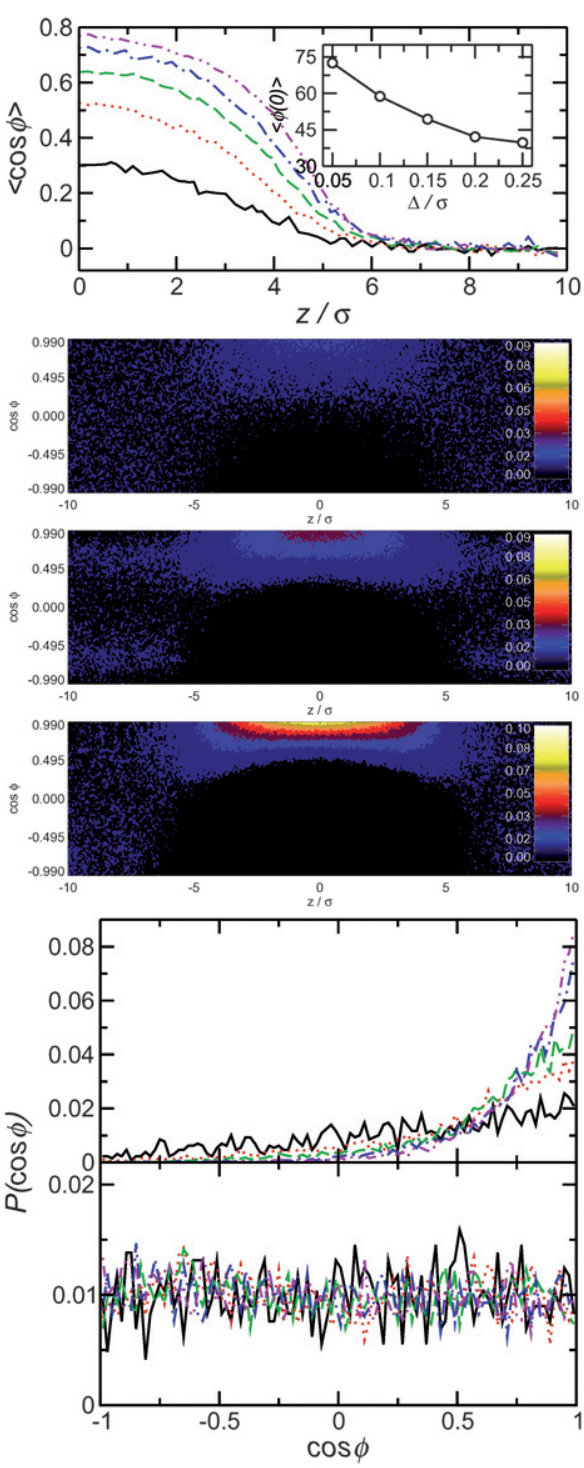

Fig. 4 (a) Free energy profiles for Janus nanoparticles with $\Delta=0$ (solid line, black), $\Delta=0.05 \sigma$ (dotted line, red), $\Delta=0.10 \sigma$ (dashed line, green), $\Delta=0.15 \sigma$ (dot-dashed line, blue), $\Delta=0.20 \sigma$ (double-dot-dashed line, magenta) and $\Delta=0.25 \sigma$ (dot-double-dashed line, cyan). (b) Detachment energy against $\Delta$. Circles (black) show the simulation barrier (open symbols free rotation, filled symbols fixed orientation) and squares (red) show results of eqn (8).

for $\Delta=0.25 \sigma$. These are significantly smaller than the the free energy difference from continuum theory (given by $2 \pi R_{c}^{2}\left[\gamma_{A P(B)}\right.$ $\left.\left.+\gamma_{B P(A)}-\gamma_{A P(A)}-\gamma_{B P(B)}\right]\right)$ which are $4.7 k_{\mathrm{B}} T$ and $16.5 k_{\mathrm{B}} T$ for $\Delta=0.05 \sigma$ and $0.25 \sigma$, respectively. This overestimation of the free energy difference likely arises due to the flat interface assumption of continuum theories. Far from the interface, the nanoparticle becomes almost completely free to rotate and the probability distribution is almost flat [Fig. 4(c)].

The effect of orientational freedom on the detachment energy may be examined by performing simulations with the nanoparticle orientation fixed $\mathbf{u}=\mathbf{z}$. Experimentally, such behaviour may be obtained by the application of an electric or magnetic field normal to the interface. As shown in Fig. 3(b), the detachment energy for the particle with fixed orientation is significantly 
higher (for $\Delta=0.25 \sigma$ the detachment energy for the fixed orientation is $10.5 k_{\mathrm{B}} T$ ) than for the freely rotating particle. $\Delta F$ for the fixed orientation is also much closer to the theoretical prediction. The remaining difference between the simulation and theoretical detachment energies may arise due to the latter's neglect of line tension. The magnitude of the line tension may be estimated from the difference between the simulation and theoretical detachment energies, and ranges from $\beta \sigma \tau=-0.10$ for $\Delta=0,{ }^{27}$ to 0.13 for $\Delta=0.25 \sigma$. The discrepancy may also arise due to an increase in the free energy of the nanoparticle caused by interactions between $A$-molecules and the $B$-philic face (and viceversa) that are neglected by the flat-interface assumption of the macroscopic models.

\subsection{Asymmetric Janus particles}

On increasing the $A$-philic portion of the nanoparticle surface (i.e. increasing $\alpha$ ) the particle becomes destabilised from the interface and moves into the $A$-rich region. This is shown by the free energy profiles [Fig. 5(a)], which become increasingly asymmetric and for $90^{\circ}<\alpha<180^{\circ}$. These interpolate between the symmetric Janus particle and homogeneous nanoparticles, as expected. As $\alpha$ increases $\Delta F_{A}$ decreases and $\Delta F_{B}$ increases. As for the symmetric particle, the detachment energies calculated from continuum theory are larger than the simulation barriers. The discrepancy decreases as $\alpha \rightarrow 180^{\circ}$ (i.e. as the nanoparticle surface becomes uniform). The position of the free energy minimum moves into the $A$-region ( $z_{c}{ }^{\min }$ decreases) as $\alpha$ increases, with $z_{c}{ }^{\min }=-0.84 \sigma,-1.79 \sigma$, and $-3.13 \sigma$ for $\alpha=112.5^{\circ}, 135^{\circ}$, and $157.5^{\circ}$ respectively. From continuum theory the position of the free energy minimum is given by $z_{c}{ }^{\min }=R_{c} \cos \alpha,{ }^{19}$ which corresponds to $-0.95 \sigma,-1.77 \sigma$, and $-2.31 \sigma$.

When $\alpha \neq 90^{\circ}$ the aligning tendency of the interface weakens and the particle exhibits greater orientational freedom. The orientational behaviour of the asymmetric Janus particles is
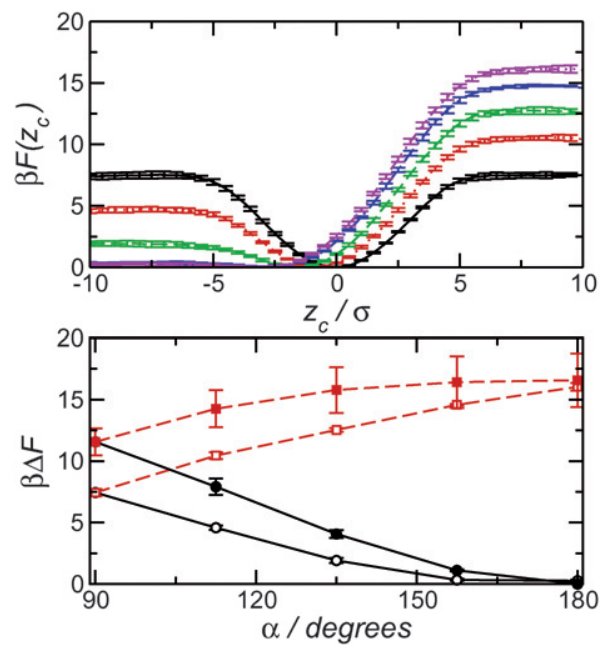

Fig. 5 (a) Free energy profiles for Janus nanoparticles with $\Delta=0.25 \sigma$ and $\alpha=90^{\circ}$ (solid line, black), $112.5^{\circ}$ (dotted line, red), $135^{\circ}$ (dashed line, green), $157.5^{\circ}$ (dot-dashed line, blue), and $180^{\circ}$ (double-dot-dashed line, magenta). (b) Free energy barriers against $\alpha$. Circles (black) show $\Delta F_{A}$ and squares (red) show $\Delta F_{B}$ (open symbols simulation, filled symbols theory). (a)

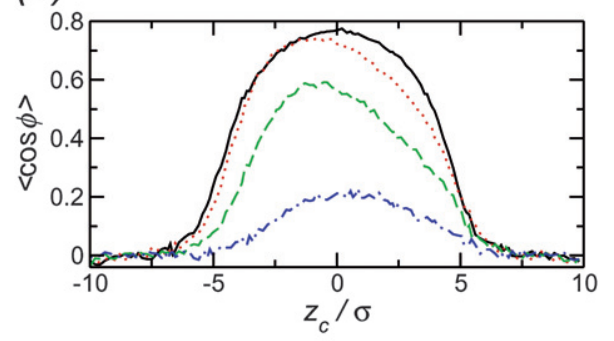

(b)
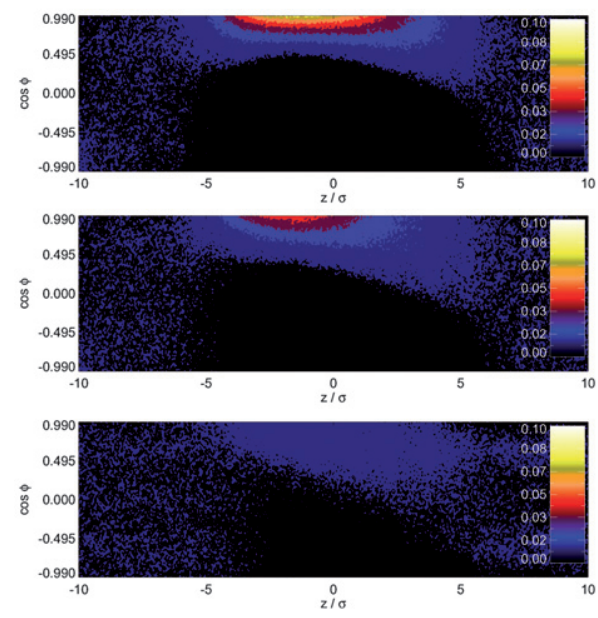

Fig. 6 (a) $\langle\cos \phi\rangle$ against nanoparticle-interface separation for Janus nanoparticle $(\Delta=0.25 \sigma)$ with $\alpha=90^{\circ}$ (solid line, black), $112.5^{\circ}$ (dotted line, red), $135^{\circ}$ (dashed line, green), and $157.5^{\circ}$ (dot-dashed line, blue). (b) Probability maps $P\left(z_{c}, \cos \phi\right)$ for $\alpha=112.5^{\circ}$ (top), $135^{\circ}$ (middle), and $157.5^{\circ}$ (bottom).

shown in Fig. 6. As $\alpha$ increases the maximum value of $\langle\cos \phi\rangle$ decreases [Fig. 6(a)]. For $\alpha=112.5^{\circ}$ and $135^{\circ}$ this is noticeably asymmetric about the interface, with the position of its maximum being in the $A$-rich region. This asymmetry is also present in $P\left(z_{c}, \cos \phi\right)$ [Fig. 6(b)]. For $z_{c}<0$ the distribution is narrower, suggesting that the particle has more orientational freedom on the $B$-rich region (which is reflected in the smaller values of $\langle\cos \phi\rangle$ for $z_{c}>0$ ). For $\alpha=157.5^{\circ}$ the interface shows only a weak orienting effect of the nanoparticle.

\section{Conclusions}

Using Monte Carlo simulations, the interaction between a Janus nanoparticle and an ideal liquid-liquid interface has been studied. In common with previous simulation work on uniform nanoparticles, ${ }^{27}$ the range of the nanoparticle-interface interaction is significantly larger than the nanoparticle radius, due to broadening of the interface by capillary waves. Some of the interaction potentials, e.g. the $\Delta=0.25 \sigma$ nanoparticle [Fig. 2(a)], decrease at large nanoparticle-interface separations. This may arise due to a draining of solvent in a wetting/dewetting transition on the particle surface and due bending of the interface. Experimental studies of colloidal particles $(\approx 1-2 \mu \mathrm{m})$ have observed an activation barrier for the attachment of particles to a liquid interface which may arise due to these effects. However, 
the height of these barriers are significantly smaller than the error bars, so the present simulations are not capable of resolving this. For the symmetric Janus nanoparticle, increasing the affinity of each hemisphere leads to stronger binding to the interface. The increase in stability with the surface tensions is smaller than predicted by macroscopic theories, which is due to their neglect of the nanoparticle's orientational freedom. Fixing the nanoparticle orientation leads to a significant increase in the nanoparticle-interface interaction strength, which suggests that the experimental stability of particles may be controlled through the application of external fields. When the size of the $A$-philic region is increased, the particle stability at the interface decreases and the orienting effect of the interface becomes weaker. The agreement between the simulation and theoretical detachment energies improves as the size of the $A$-philic region increases, as the surface term in the free energy becomes the dominant contribution.

It is worth reflecting on the relationship between the simulated system and those encountered in experiments. The fluid structure at the interface is likely to be quite different than in experimental systems, particular for water or other hydrogen-bonding liquids, which may give rise to more complex potentials of mean force than presented here. Simulations using more sophisticated models are likely to be able reproduce these more complex interactions. The detachment energies, which largely derive from changes to interface and surface free energies are, however, likely to be similar. Assuming a molecular size $\sigma \approx 0.3 \mathrm{~nm}$ gives an interfacial tension $\gamma_{A B} \approx 6 \mathrm{mN} \mathrm{m}^{-1}$, which is smaller than but comparable to commonly used liquid mixtures (e.g. $\gamma_{A B}=36 \mathrm{mN} \mathrm{m}^{-1}$ for watertoluene). ${ }^{46}$ Similarly, mapping the nanoparticle-solvent surface tensions gives $\Delta \gamma \approx 8 \mathrm{mN} \mathrm{m}^{-1}$ compared to $\Delta \gamma \approx 25 \mathrm{mN} \mathrm{m}^{-1}$ for TOPO-covered nanoparticles at the water-toluene interface. This suggests that, despite the simplicity of the model studied, the results here are relevant to the behaviour of experimental systems. In particular, the reduction in the stability of Janus particles due to their orientational freedom is likely to be important in the adhesion and self-assembly of Janus nanoparticles on liquid interfaces.

\section{Appendix}

\section{Acceptance rules for Monte Carlo moves}

The acceptance rules for the different MC moves are:

- Translations are rejected if (i) any overlaps (between solvent particles or between the nanoparticle and solvent) occur in the new configuration or (ii)

$$
\exp (-\beta \Delta w)<\zeta \quad \text { (Metropolis criteria) }
$$

where $\Delta w=w\left(z_{\text {new }}\right)-w\left(z_{\text {old }}\right)$ is the difference between the weight function at the new and old configurations and $\zeta$ is a random number between 0 and 1 . Note that changes in the solvent particle coordinates may lead to changes in the interface position, thus changing the nanoparticle-interface separation. The maximum translation size is chosen to give an acceptance rate of approximately $40 \%$ for solvent particles and $10 \%$ for nanoparticles.
-Nanoparticle rotations and flips are rejected if any overlaps occur in the new configuration (the nanoparticle-interface separation is unchanged on rotational moves).

-Solvent particle identity swaps are rejected if (i) overlaps occur in the new configuration or (ii) the Metropolis criteria on the weight function eqn (9) is failed.

-Solvent particle insertions are rejected if (i) the inserted particle overlaps with an existing particle, (ii) the Metropolis criteria on the weight function is failed, or (iii)

$$
\exp \left\{-\beta\left[\ln \left(\frac{N_{i}+1}{V}\right)-\mu\right]\right\}<\zeta
$$

where $N_{i}, i=A, B$ is the number of particles of component $i{ }^{47}$

-Solvent particle deletions are rejected if (i) the Metropolis check on the weight function is failed or (ii)

$$
\exp \left\{-\beta\left[\mu+\ln \left(\frac{V}{N_{i}}\right)\right]\right\}<\zeta
$$

\section{Theoretical prediction of Janus particle detachment energy}

Assuming a flat interface and neglecting line tension, the detachment energy of a Janus particle from a liquid interface may be estimated purely from the changes in the surface free energies. ${ }^{19,43}$ The equilibrium immersion depth $z_{c}{ }^{\text {eq }}=\cos$ $\beta$ depends on the relative magnitudes of the contact angles cos $\theta_{A P}=\left(\gamma_{A P(B)}-\gamma_{A P(A)}\right) / \gamma_{A B}$ and $\cos \theta_{B P}=\left(\gamma_{B P(B)}-\gamma_{A P(B)}\right) / \gamma_{A B}$ and the relative areas of the $A$ and $B$-philic regions (parameterised by $\alpha$ ). As $\gamma_{A P(A)}=\gamma_{B P(B)}$ and $\gamma_{B P(A)}=\gamma_{A P(B)}$, for the particles studied in this work, $\cos \theta_{A P}=-\cos \theta_{B P}$. In all cases studied in this work the condition $\theta_{A}<\alpha<\theta_{B}$; for the symmetric, $\alpha=90^{\circ}$, case (section 3.2) $\theta_{A}<90^{\circ}$ and $\theta_{B}>90^{\circ}$, while for the asymmetric particles (section 3.3) the $\left|\gamma_{A P}-\gamma B P\right|>\gamma_{A B}$, so $\theta_{A}<0$ and $\theta_{B}>180^{\circ}$ and $\theta_{A}<\alpha<\theta_{B}$ for all values of $\alpha$. This condition then implies that $\beta=\alpha{ }^{19}$

For a particle at the equilibrium position the surface free energy is then

$$
\begin{gathered}
F_{S}^{\mathrm{int}}=-\pi R_{c}^{2} \sin ^{2} \beta \gamma_{A B}+2 \pi R_{c}^{2}(1-\cos \beta) \gamma_{A P(A)} \\
+2 \pi R_{c}^{2}(1+\cos \beta) \gamma_{B P(B)}
\end{gathered}
$$

where the terms on the right hand side correspond to the decrease in the $A B$ interfacial area $\left(\pi R_{c}^{2} \sin ^{2} \beta\right)$, the interaction between the $A$-philic patch on the particle and the $A$-component, and the interaction between the $B$-philic patch on the particle and the $B$-component. When the particle is in the bulk of the $A$ component the surface free energy is then

$$
F_{\mathrm{s}}{ }^{A}=2 \pi R_{c}{ }^{2}(1-\cos \beta) \gamma_{A P(A)}+2 \pi R_{c}^{2}(1+\cos \beta) \gamma_{B P(A)}
$$

which corresponds to the interaction between the $A$ and $B$-philic regions with the $A$-component. Similarly the surface free energy in the bulk of the $B$-component is

$$
F_{\mathrm{s}}{ }^{B}=2 \pi R_{c}^{2}(1-\cos \beta) \gamma_{A P(B)}+2 \pi R_{c}^{2}(1+\cos \beta) \gamma_{B P(B)}
$$

The detachment energies eqn (8) are then found by subtraction of eqn (12) from eqn (13) and eqn (14) and recalling that $\alpha=\beta$. 


\section{Acknowledgements}

The authors wish to thank Martin Oettel (Mainz) for a critical reading and helpful comments on this manuscript. This work was funded by UK EPSRC and computational resources for this work were provided by the Centre for Scientific Computing, University of Warwick.

\section{References}

1 W. Ramsden, Proc. R. Soc. London, Ser. A, 1903, 72, 156-164.

2 S. U. Pickering, J. Chem. Soc. Trans., 1907, 91, 2001-2021.

3 F. Bresme and M. Oettel, J. Phys.: Condens. Matter, 2007, 19(41), 413101.

4 W. H. Binder, Angew. Chem., Int. Ed., 2005, 44(33), 5172-5175.

5 A. Böker, J. He, T. Emrick and R. P. Russell, Soft Matter, 2007, 3, $1231-1248$

6 P. Arumugam, D. Pattra, B. Samanta, S. S. Agasta, C. Subramani and V. M. Rotello, J. Am. Chem. Soc., 2008, 130, 10046-10047.

7 R. P. Sear, S. W. Chung, G. Markovich, W. M. Gelbart and J. R. Heath, Phys. Rev. E: Stat., Nonlinear, Soft Matter Phys., 1999, 59, R6255-R6258.

8 G. A. Ozin and A. Arsenault, Nanochemistry: A chemical approach to nanomaterials, RSC, Cambridge, 2005.

9 B. P. Binks and K. Murakami, Nat. Mater, 2006, 5(11), 865-869.

10 P. S. Clegg, J. Phys.: Condens. Matter, 2008, 20(11), 113101.

11 S. A. F. Bon and P. J. Colver, Langmuir, 2007, 23(16), 8316-8322.

12 P. J. Colver, C. A. L. Colard and S. A. F. Bon, J. Am. Chem. Soc., 2008, 130(50), 16850-16851.

13 A. Walther and A. H. E. Müller, Soft Matter, 2008, 4(4), 663-668.

14 H. J. Hektor and K. Scholtmeijer, Curr. Opin. Biotechnol., 2005, 16(4), 434-439.

15 P. Akcora, H. Liu, S. K. Kumar1, J. Moll, Y. Li, B. C. Benicewicz, L. S. Schadler, D. Acehan, A. Z. Panagiotopoulos, V. Pryamitsyn, V. Ganesan, J. Ilavsky, P. Thiyagarajan, R. M. Colby and J. F. Douglas, Nat. Mater., 2009, 8(4), 354-359.

16 M. S. Nikolic, C. Olsson, A. Salcher, A. Kornowski, A. Rank, R. Schubert, A. Frömsdorf, H. Weller and S. Förster, Angew. Chem., Int. Ed., 2009, 48(15), 2752-2754.

17 P. Pieranski, Phys. Rev. Lett., 1980, 45(7), 569-572.

18 R. Aveyard and J. H. Clint, J. Chem. Soc., Faraday Trans., 1996, 92(1), 85-89.

19 B. P. Binks and P. D. I. Fletcher, Langmuir, 2001, 17(16), 4708-4710.

20 Y. Nonomura, S. Komura and K. Tsujii, Langmuir, 2004, 20(26), $11821-11823$.
21 Y. K. Takahara, S. Ikeda, S. Ishino, K. Tachi, K. Ikeue, T. Sakata, T. Hasegawa, H. Mori, M. Matsumura and B. Ohtani, J. Am. Chem. Soc., 2005, 127(17), 6271-6275.

22 N. Glaser, D. J. Adams, A. Böker and G. Krausch, Langmuir, 2006, 22(12), 5227-5229.

23 A. Centrone, E. Penzo, M. Sharma, J. W. Myerson, A. W. Jackson, N. Marzari and F. Stellacci, Proc. Natl. Acad. Sci. U. S. A., 2008, 105(29), 9886-9891.

24 F. Bresme and N. Quirke, Phys. Rev. Lett., 1998, 80, 3791-3794.

25 F. Bresme and N. Quirke, J. Chem. Phys., 1999, 110, 3536-3547.

26 F. Bresme and N. Quirke, Phys. Chem. Chem. Phys., 1999, 1, 21492155.

27 D. L. Cheung and S. A. F. Bon, Phys. Rev. Lett., 2009, 102(6), 066103.

28 H. Lehle and M. Oettel, J. Phys.: Condens. Matter, 2008, 20(40), 404224.

29 F. Bresme, H. Lehle and M. Oettel, J. Chem. Phys., 2009, 130(21), 214711.

30 B. Widom and J. S. Rowlinson, J. Chem. Phys., 1970, 52, 1670.

31 A. Malijevský and S. Sokoł owski, J. Chem. Phys., 2007, 126(10), 106101.

32 E. de Miguel, N. G. Almarza and G. Jackson, J. Chem. Phys., 2007, 127(3), 034707, 03.

33 Y. Djikaev, J. Chem. Phys., 2008, 128(1), 014712.

34 F. G. Wang and D. P. Landau, Phys. Rev. Lett., 2001, 86, 2050-2053.

35 A. M. Ferrenberg and R. H. Swendsen, Phys. Rev. Lett., 1989, 63(12), $1195-1198$.

36 F. Calvo, Mol. Phys., 2002, 100(21), 3421-3427.

37 E. B. Kim, R. Faller, Q. Yan, N. L. Abbot and J. J. de Pablo, J. Chem. Phys., 2002, 117, 7781-7787.

38 D. L. Cheung, L. Anton, M. P. Allen and A. J. Masters, Phys. Rev. E: Stat., Nonlinear, Soft Matter Phys., 2006, 73(6), 061204.

39 R. E. Belardinelli and V. D. Pereyra, J. Chem. Phys., 2007, 127(18), $184105,01$.

40 D. J. Earl and M. W. Deem, J. Phys. Chem. B, 2005, 109, 6701-6704.

41 A. Laio and F. L. Gervasio, Rep. Prog. Phys., 2008, 71(12), 126601.

42 U. O. M. Vázquez, W. Shinoda, P. B. Moore, C.-C. Chiu and S. O. Neilsen, J. Math. Chem., 2009, 45(1), 161-174.

43 S. Jiang and S. Granick, J. Chem. Phys., 2007, 127(16), 161102.

44 J. R. Link and M. J. Sailor, Proc. Natl. Acad. Sci. U. S. A., 2003, 100(19), 10607-10610.

45 D. J. Adams, S. Adams, J. Melrose and A. C. Weaver, Colloids Surf., A, 2008, 317(1-3), 360-365.

46 Y. Lin, H. Skaff, T. Emrick, A. D. Dinsmore and T. P. Russell, Science, 2003, 299(5604), 226-229.

47 D. Frenkel and B. Smit, Understanding molecular simulation: from algorithms to applications, Academic Press, San Diego, 2nd edn, 2002. 